\title{
Reusable venesection tourniquets: a potential source of hospital transmission of multiresistant organisms
}

\section{Angie N Pinto MB BS, BSc, MPHTM Microbiology Registrar \\ Thuy Phan BSc, Technical Officer \\ Gabriela Sala BSc, Hospital Scientist \\ Elaine Y L Cheong FRACP, FRCPA, Infectious Diseases Physician and Microbiologist \\ Steven Siarakas BSc, PhD, Senior Hospital Scientist \\ Thomas Gottlieb FRACP, FRCPA, Infectious Diseases Physician and Microbiologist \\ Department of Microbiology and Infectious Diseases, Concord Repatriation Genera \\ Hospital, Sydney, NSW. \\ pinto.angie@gmail.com}

MJA 2011; 195: 276-279 doi: 10.5694/mjall.10333

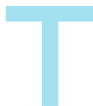

he hospital environment can be a source of transmission of multiresistant organisms (MROs). Hospital infection control policies attempt to minimise crosstransmission of MROs, which include methicillin-resistant Staphylococcus aureus (MRSA), vancomycin-resistant enterococci (VRE), and Enterobacteriaceae harbouring transmissible extended-spectrum $\beta$-lactamases (ESBLs) and metallo- $\beta$-lactamases (MBLs). Surfaces such as keyboards, ${ }^{1,2}$ stethoscopes, ${ }^{3-6}$ ties, $^{7-11}$ lanyards $^{12}$ and tourniquets ${ }^{1,13-15}$ have the potential to act as fomites and can harbour pathogenic microorganisms.

Reusable venesection tourniquets are often used consecutively on multiple patients without disinfection between uses. Current Australian health care guidelines suggest cleaning of these non-critical items with a neutral detergent on a regular basis. ${ }^{16}$ However, the required frequency of cleaning is not specified, nor whether this would prevent transmission of MROs to patients. Previous studies have indicated varying rates of MRO colonisation of reusable tourniquets, and differ based on the sensitivity of the culture method used. ${ }^{17}$ We performed this study to determine the prevalence of $\mathrm{MRO}$ colonisation of reusable venesection tourniquets in a Sydney teaching hospital using a sensitive enrichment method.

\section{Methods}

\section{Hospital setting}

The study was conducted at Concord Hospital, a 503-bed metropolitan teaching hospital in Sydney. Random sampling and testing of 100 reusable tourniquets took place over a 10-week period between September and November 2010. Tourniquets were randomly collected from locations throughout the hospital, including general wards, ambulatory care areas (defined as outpatient clinics, the

\begin{abstract}
Abstrac i
Objective: To determine the prevalence of multiresistant organism (MRO) colonisation of reusable venesection tourniquets.

Design and setting: A prospective study in a tertiary hospital to collect and analyse reusable venesection tourniquets for the presence of MROs methicillin-resistant Staphylococcus aureus (MRSA), vancomycin-resistant enterococci (VRE), and extended-spectrum $\beta$-lactamase and metallo- $\beta$ lactamase-producing Enterobacteriaceae - using a sensitive enrichment method. Tourniquets were collected and tested during a 10-week period between September and November 2010.
\end{abstract}

Main outcome measure: Prevalence of MRO colonisation of tourniquets. Results: The overall colonisation rate of 100 tourniquets randomly collected from general wards, ambulatory care areas and critical care areas was $78 \%$. MROs were isolated from 25 tourniquets collected from a variety of hospital locations, including general wards, the intensive care unit, burns unit and anaesthetic bay. MRSA was isolated from 14 tourniquets and VRE from 19; both MRSA and VRE were isolated from nine tourniquets. There were no microorganisms isolated from 22 tourniquets.

Conclusion: Reusable tourniquets can be colonised with MROs and may be a potential source of transmission of MROs to hospitalised patients.

blood collection unit, doctors' offices and the emergency department) and critical care areas (defined as the operating theatre, intensive care unit [ICU] and burns unit).

This study was organised by the hospital infection control committee as an investigation into tourniquet contamination and disinfection. As there were no patients involved and no patient specimens collected, ethics approval was not sought.

\section{Microbiology}

Reusable tourniquets were collected and immediately placed into a polyethylene specimen bag, labelled and transferred to the laboratory. At twice weekly intervals, tourniquets were immersed in an enrichment medium (Brain Heart Infusion Broth; Oxoid Australia, Adelaide, SA) and incubated overnight. Fluid from the broth was then subcultured onto a variety of agar media: horse-blood agar (Columbia HBA; Oxoid), MacConkey agar (Oxoid), and selective agar media for the detection of MRSA (MRSASelect; Bio-Rad, Sydney, NSW), VRE (chromID VRE; bioMérieux, Sydney, NSW) and resistant gram-negative bacteria including ESBL- and MBL- producing organisms (Brilliance ESBL Agar; Oxoid). Significant isolates were identified, and resistance gene testing was performed for the confirmation of MRSA, VRE and MBL resistance.

Growth of isolates from broth enrichment was recorded, and classified as: environmental organisms or bacteria of low pathogenic potential; "potentially significant" bacteria; and MROs (defined as MRSA, VRE, and MBL- and ESBL-producing Enterobacteriaceae). We typed VRE isolates using a DiversiLab rep-PCR system (bioMérieux). Tourniquets that tested positive for MROs or other potentially significant organisms were discarded.

\section{Results}

Tourniquet collection data are summarised in Box 1. The majority of tourniquets were collected from areas where they are frequently used, such as the blood collection unit $(n=7)$, and from general medical and surgical wards. Bacteria were isolated from tourniquets collected in every week of the study period. The overall bacterial colonisation rate of the 100 tourniquets was $78 \%$. There was no bacterial growth from 22 tourniquets, and 
17 grew only environmental organisms or bacteria of low pathogenic potential (coagulase-negative staphylococci and/or Bacillus spp).

Microbial colonisation data are summarised in Box 2. Many tourniquets were colonised with more than one organism. Ten grew potentially significant gram-positive organisms (methicillin-sensitive Staphylococcus aureus or Enterococcus spp), and 38 grew potentially significant gramnegative organisms (Pseudomonas spp and/or Enterobacteriaceae).

MROs were found on 25 tourniquets; however, three had been collected from $\mathrm{MRO}$ isolation rooms. An IMP-4 MBL-positive Enterobacter cloacae and an ESBL-positive E. cloacae were each isolated from a single tourniquet. MRSA was isolated from 14 tourniquets. VRE was isolated from 19 tourniquets: $v a n B$-positive Enterococcus faecium from 18, and vanA-positive Enterococcus faecalis from one. Nine tourniquets isolated both MRSA and VRE, and 24 grew one or the other of these. Typing of the 18 vanB-positive isolates demonstrated five VRE clusters (Box 3). There was no apparent association between clusters of enterococci and hospital location.

Six of nine tourniquets collected from the ICU throughout the study period grew at least one MRO, although two had been used on patients known to be colonised with MRSA. MROs were isolated from tourniquets collected in most weeks of the study period (Box 1) from various hospital locations, including general wards, the ICU, burns unit, operating theatre anaesthetic bay, and the blood collection unit. The ICU had the highest rate of MRO colonisation $(67 \%$ [6/9] v 23\% [15/64] in wards and 13\% [3/23] in ambulatory care areas).

\section{Discussion}

We found that $61 \%$ of reusable tourniquets were colonised with bacterial species that would not be considered normal upper-limb skin flora and that can be associated with hospitalacquired bacteraemia. A quarter of randomly collected tourniquets yielded an MRO. If a single patient $\mathrm{MRO}$ transmission is perceived to be

1 Tourniquets sampled from each location, by most clinically significant category of bacteria isolated

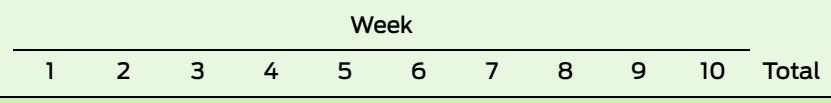

\begin{tabular}{|c|c|c|c|c|c|c|c|c|c|c|c|}
\hline Wards & & & & & & & & & & & \\
\hline No bacterial growth & 0 & 1 & 2 & 3 & 1 & 1 & 3 & 2 & 0 & 0 & 13 \\
\hline $\begin{array}{l}\text { Environmental or low pathogenic } \\
\text { potential organisms }\end{array}$ & 0 & 2 & 0 & 2 & 0 & 2 & 1 & 1 & 2 & 1 & 11 \\
\hline Potentially significant non-MROs & 0 & 5 & 3 & 3 & 1 & 5 & 3 & 3 & 2 & 0 & 25 \\
\hline MROs & 0 & 0 & 1 & 3 & 1 & 4 & 4 & 1 & 1 & 0 & 15 \\
\hline \multicolumn{12}{|l|}{ Ambulatory care areas* } \\
\hline No bacterial growth & 0 & 1 & 1 & 0 & 4 & 0 & 0 & 2 & 0 & 0 & 8 \\
\hline $\begin{array}{l}\text { Environmental or low pathogenic } \\
\text { potential organisms }\end{array}$ & 2 & 0 & 0 & 0 & 0 & 0 & 1 & 0 & 0 & 0 & 3 \\
\hline Potentially significant non-MROs & 0 & 3 & 0 & 0 & 4 & 2 & 0 & 0 & 0 & 0 & 9 \\
\hline MROs & 1 & 1 & 0 & 0 & 0 & 1 & 0 & 0 & 0 & 0 & 3 \\
\hline \multicolumn{12}{|l|}{ Critical care areas ${ }^{\dagger}$} \\
\hline No bacterial growth & 0 & 0 & 0 & 0 & 0 & 0 & 0 & 1 & 0 & 0 & 1 \\
\hline $\begin{array}{l}\text { Environmental or low pathogenic } \\
\text { potential organisms }\end{array}$ & 0 & 1 & 0 & 0 & 0 & 0 & 1 & 0 & 0 & 1 & 3 \\
\hline Potentially significant non-MROs & 0 & 1 & 0 & 0 & 0 & 0 & 0 & 1 & 0 & 0 & 2 \\
\hline MROs & 0 & 1 & 3 & 1 & 1 & 0 & 0 & 0 & 1 & 0 & 7 \\
\hline Total tourniquets & 3 & 16 & 10 & 12 & 12 & 15 & 13 & 11 & 6 & 2 & 100 \\
\hline
\end{tabular}

MROs = multiresistant organisms. * Emergency department, outpatient clinics, blood collection unit and doctors' offices. †Operating theatre, intensive care unit and burns unit.

\section{Microbial colonisation of tourniquets*}

\begin{tabular}{lcccc} 
Organism & Wards & Ambulatory care & Critical care & Total \\
\hline Total tourniquets collected & 64 & 23 & 13 & 100 \\
Environmental or low pathogenic potential & 10 & 2 & 1 & 13 \\
Coagulase-negative staphylococci & 37 & 9 & 8 & 54 \\
Bacillus spp & & & 0 & 9 \\
Potentially significant non-MROs & 7 & 2 & 0 & 1 \\
Enterococcus spp & 1 & 0 & 2 & 26 \\
Methicillin-sensitive Staphylococcus aureus & 15 & 9 & 0 & 18 \\
Enterobacteriaceae & 12 & 6 & & 14 \\
Pseudomonas spp & & & 6 & 19 \\
MROs & 6 & 2 & 6 & 1 \\
Methicillin-resistant Staphylococcus aureus & 10 & 3 & 1 & 1 \\
Vancomycin-resistant enterococci & 0 & 0 & 0 & \\
Extended-spectrum $\beta$-lactamases & 1 & 0 & & \\
Metallo- $\beta$-lactamases & & & & \\
\hline
\end{tabular}

MROs = multiresistant organisms. $*$ Many tourniquets had mixed bacterial growth.

an avoidable patient care outcome, then any reuse of $\mathrm{MRO}$-colonised tourniquets may present an unacceptable risk.

It is estimated that around $6 \%$ of hospitalised patients will acquire an infection during their admission, leading to increased length of stay, further treatment and higher overall cost. ${ }^{18}$ To what extent tourniquets contribute to colonisation, and possibly bacteraemia, is uncertain. MRO colonisation of tourniquets may reflect the burden of MROs in the wider hospital environment and provide a measurable index of the level and quality of hospital environmental hygiene. Tourniquets may have higher potential for MRO transfer than other fomites as they are applied under pressure against the patient's skin. They are also placed in close proximity to vascular access sites, and any skin colonisation could lead to preventable complications or health care-associated infections, such as phlebitis or 
3 Typing of vancomycin-resistant enterococci isolates from tourniquets*

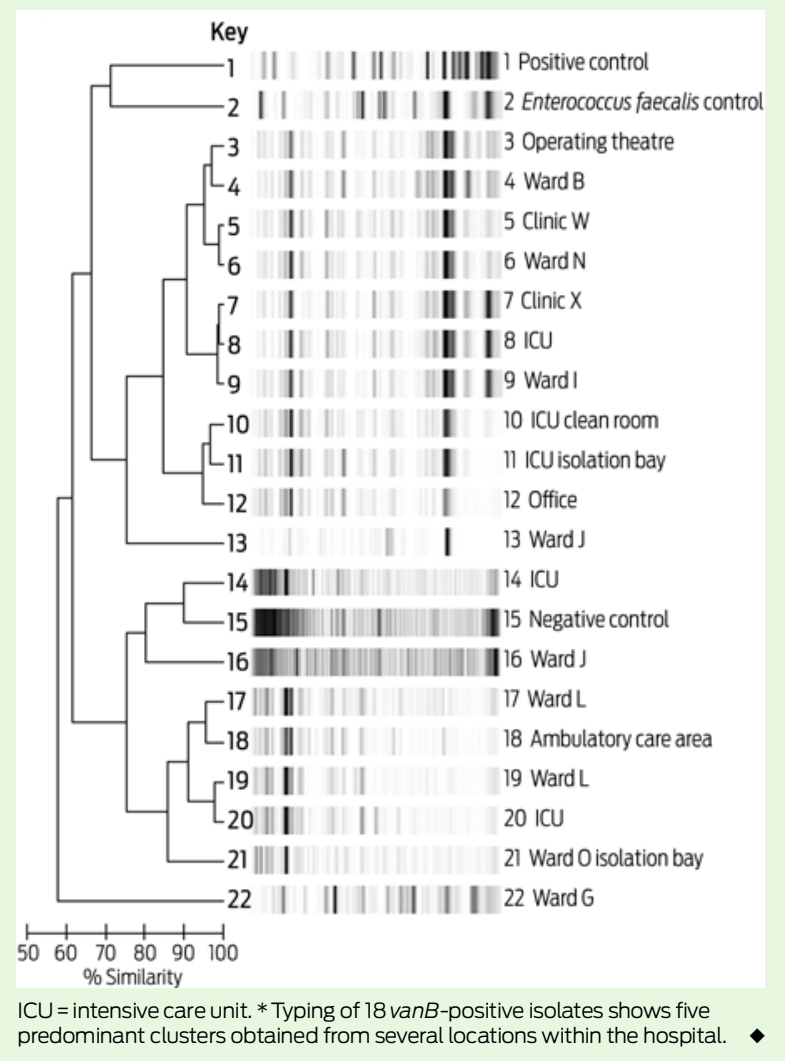

cannula site infections. It is untenable that patients are exposed to potentially virulent pathogens on reused equipment.

While disposable tourniquets are readily available, their use is not universal due to perceived difficulties in application and patient discomfort. However, a study found that $85 \%$ of patients found disposable tourniquets at least as good as reusable tourniquets, and $95 \%$ of doctors found them as easy to use. ${ }^{19}$ With adequate training provided, and at a cost of about 50 cents per unit (BD, Sydney, NSW), disposable tourniquets are a viable alternative for preventing acquisition of MROs in the hospital environment. However, there is currently no supporting evidence that introducing disposable tourniquets reduces hospital MRO acquisition rates. Moreover, such a measure should be one element of a bundle of infection control measures implemented to improve hospital environmental hygiene, and hence it may be difficult to measure its contribution to reduced MRO rates.
While previous studies have demonstrated MRSA colonisation rates ranging between 0 and $42 \%,{ }^{16}$ none have reported rates of VRE colonisation. We also identified colonisation by multiresistant gram-negative organisms with transmissible $\beta$-lactamase enzymes, including IMP-4. The presence of such enzymes can result in infections that are virtually untreatable with available antibiotics. These have previously been shown to be transmitted readily throughout the hospital environment. ${ }^{20}$

We found that the highest rate of colonisation of MROs was in the ICU. ICUs are recognised as hospital sites with high throughput of patients and staff, and with resultantly higher acquisition rates and difficulty in controlling transmission of MROs. We found that VRE clusters isolated from tourniquets in the ICU did not appear to be clonally related, reflecting the complex pattern of movement of staff, patients and tourniquets within the hospital. Tourniquets in the ICU in this study were allocated for single patient use, which demonstrates that MRO colonisation was not necessarily due to reuse, but that deficiencies in hospital environmental hygiene are likely to contribute to ongoing $\mathrm{MRO}$ colonisation of tourniquets in the ICU. Although several tourniquets were obtained from isolation rooms that accommodated patients already colonised with an MRO, and may therefore reflect the patient's own flora, the majority had been used on patients whose screening had not identified MRO colonisation.

MROs may remain viable in the environment for a long time, as demonstrated by an MRO-colonised tourniquet (collected from an office) that had not been used for several months. When tourniquets are carried from ward to ward by hospital staff and used repeatedly, they may become a "sleeper" mechanism for unrecognised hospital MRO transmission. Of concern were the nine tourniquets that were colonised with both MRSA and VRE. This probably reflects a baseline prevalence of co-colonisation of $20 \% .^{21}$

A limitation of our study is that data on tourniquet use could not be collected. Previous studies have surveyed health care personnel about hand hygiene practices and glove and tourniquet use. ${ }^{22}$ The tourniquets sampled in our study were shared among multiple users and may have been used in many different hospital wards. However, this reflects the hospital's day-today practice of tourniquet use. There was no way of tracking how often the tourniquet had been used, or where MRO acquisition had occurred. A British study demonstrated that contamination of tourniquets could be attributed to the user's hands rather than the patient's skin. ${ }^{23}$ We hypothesise that MRO colonisation of tourniquets can also be acquired from the surrounding hospital environment. The random hospital locations of the VRE clusters we recovered lends some anecdotal support to this hypothesis. A study examining colonisation of surfaces where tourniquets are stored may resolve this issue.

In this study, we did not culture for Clostridium difficile, which requires specialised media and incubation conditions to detect. C. difficile-associated diarrhoea can cause significant morbidity and mortality in hospitalised patients, and it is known that its spores may survive for a long time in the environment.

Previous studies ${ }^{16}$ have determined the limit of detection and performed semi-quantitative bacterial counts for MROs. ${ }^{23}$ Our study used a broth enrichment method, which may have increased sensitivity compared with methods used in previous studies, and we felt it was sufficient to demonstrate viability of bacteria from tourniquets using this method.

Reducing the burden of hospitalassociated infections is being addressed through multifaceted approaches such as hand hygiene and antimicrobial stewardship programs. As reusable tourniquets are frequently colonised with MROs and may be a source of cross-transmission, the burden of MRO colonisation from the hospital environment also needs to be considered. With current high prevalence rates of MROs, continued use of reusable tourniquets may not be justified in the hospital setting.

Acknowledgements: We thank Dr Amrita Ronnachit for assistance with tourniquet collection, and Dr Tony Pavic for assistance with VRE typing.

Competing interests: No relevant disclosures.

Received 23 Mar 2011, accepted 29 Jun 2011. 
1 Fellowes C, Kerstein R, Clark J, Azadian BS. MRSA on tourniquets and keyboards. J Hosp Infect 2006; 64: 86-88.

2 Simmons N. Computer keyboards and the spread of MRSA. J Hosp Infect 2006; 64: 88.

3 Marinella MA, Pierson C, Chenoweth C. The stethoscope. A potential source of nosocomial infection? Arch Intern Med 1997; 157: 786-790.

4 Bernard L, Kereveur A, Durand D, et al. Bacterial contamination of hospital physicians' stethoscopes. Infect Control Hosp Epidemiol 1999; 20: 626-628.

5 Varghese D, Patel H. Hand washing. Stethoscopes and white coats are sources of nosocomial infection. BMJ 1999; 319: 519.

6 Kennedy KJ, Dreimanis DE, Beckingham WD, Bowden FJ. Staphylococcus aureus and stethoscopes [letter]. Med J Aust 2003; 178: 468.

7 Dixon M. Neck ties as vectors for nosocomial infection. Intensive Care Med 2000; 26: 250.

8 Ditchburn I. Should doctors wear ties? J Hosp Infect 2006; 63: 227-228.

9 Day M. Doctors are told to ditch "disease spreading” neckties [news]. BMJ 2006; 332: 442.

10 Biljan MM, Hart CA, Sunderland D, et al. Multicentre randomised double blind crossover trial on contamination of conventional ties and bow ties in routine obstetric and gynaecological practice. BMJ 1993; 307: 1582-1584.

11 Steinlechner C, Wilding G, Cumberland N. Microbes on ties: do they correlate with wound infection? Bull R Coll Surg Engl 2002; 84: 307-309.

12 Kotsanas D, Scott C, Gillespie EE, et al. What's hanging around your neck? Pathogenic bacteria on identity badges and lanyards. Med J Aust 2008; 188: 5-8.

13 Golder M, Chan CLH, O'Shea S, et al. Potential risk of cross-infection during peripheral-venous access by contamination of tourniquets. Lancet 2000; 355: 44.

14 Berman DS, Schaefler S, SimberkoffMS, Rahal JJ. Tourniquets and nosocomial methicillin-resistant Staphylococcus aureus infections. N Engl J Med 1986; 315: 514-515.

15 Ahmed SM, Ahmad R, Case R, Spencer RF. A study of microbial colonisation of orthopaedic tourniquets. Ann R Coll Surg Engl 2009; 91: 131-134.

16 National Health and Medical Research Council. Australian guidelines for the prevention and control of infection in healthcare. Canberra: NHMRC, 2010. http://www.nhmrc.gov.au/_files nhmrc/publications/attachments/cd33_ complete.pdf (accessed Jul 2011).
17 Hensley DM, Krauland KJ, McGlasson DL. Acinetobacter baumannii and MRSA contamination on reusable phlebotomy tourniquets. Clin Lab Sci 2010; 23: 151-156.

18 Spelman DW. 2: Hospital-acquired infections [MJA Practice Essentials]. Med J Aust 2002; 176: 286-291.

19 Kerstein RL, Fellowes C. Novel fit for purpose single use tourniquet: best of both worlds. J Med Eng Technol 2009; 33: 475-480.

20 Peleg AY, Franklin C, Bell JM, Spelman DW. Dissemination of the metallo- $\beta$-lactamase gene blalMP-4 among gram-negative pathogens in a clinical setting in Australia. Clin Infect Dis 2005; 41: 1549-1556.

21 Reyes K, Malik R, Moore C, et al. Evaluation of risk factors for coinfection or cocolonization with vancomycin-resistant enterococcus and methicillin-resistant Staphylococcus aureus. J Clin Microbiol 2010; 48: 628-630.

22 Sacar S, Turgut H, Kaleli I, et al. Poor hospital infection control practice in hand hygiene, glove utilization, and usage of tourniquets. Am J Infect Control 2006; 34: 606-609.

23 Leitch A, McCormick I, Gunn I, Gillespie T. Reducing the potential for phlebotomy tourniquets to act as a reservoir for meticillin-resistant Staphylococcus aureus. J Hosp Infect 2006; 63: 428-431. 